\title{
Revista Chilena de Pediatría 1999-2009: Logros de un Período
}

\author{
FRANCISCO CANO SCH. ${ }^{1}$, FRANCISCA UGARTE P. ${ }^{2}$ \\ 1. Director-Editor, Revista Chilena de Pediatría. \\ 2. Subdirectora-Coeditora, Revista Chilena de Pediatría.
}

Hace 10 años los editores recibimos del Dr. Carlos Toro la Revista Chilena de Pediatría, incorporándose a uno de los proyectos que ha contribuido en forma decisiva a la difusión de revistas biomédicas de Latinoamérica y el $\mathrm{Ca}$ ribe, el proyecto SciELO (Scientific Electronic Library on Line). El proyecto SciELO es una iniciativa de FAPESP (Fundación de Apoyo a la Investigación del Estado de São Paulo) y de BIREME (Centro Latinoamericano y del Caribe de Información en Ciencias de la Salud), que fue iniciado en Brasil en el año 1997 y cuenta en la actualidad con la participación de ocho países: Argentina, Brasil, Chile, Colombia, Cuba, España, Portugal y Venezuela; incluye también los sitios SciELO en Ciencias Sociales y Salud Pública y se está implementando en otros cinco países: Costa Rica, México, Paraguay, Perú y Uruguay. Revista Chilena de Pediatría recibe en la actualidad más de 2 millones de visitas anuales, siendo la segunda revista más visitada de SciELO Chile.

La gran visibilidad de Revista Chilena de Pediatría en SciELO, nos llevó a proponer al Directorio de la época desarrollar un proyecto de digitalización de la colección histórica de Revista Chilena Pediatría y su incorporación a la base SciELO. El proceso no estuvo libre de dificultades, ya que la metodología SciELO no era compatible con el formato de nuestros primeros números que carecían de resúmenes y palabras claves, contenían información de re- uniones clínicas y las opiniones de los primeros pediatras de nuestro país. Con el financiamiento de SOCHIPE, el apoyo de CONYCIT, en especial de Ana María Prat, fueron resolviéndose cada uno de los problemas. Gracias a este proyecto Revista Chilena de Pediatría tiene disponibles 409 números que cubren desde 1940 hasta la fecha, siendo la única revista que ha incorporado en forma completa su colección histórica.

Desde hace algunos años, Revista Chilena de Pediatría, al igual que los órganos editoriales de alto impacto local, han vivido la experiencia de un progresivo aumento en la cantidad de manuscritos que se reciben, incluyendo publicaciones de diferentes países de América Latina. Esta realidad nos llevó a plantear la necesidad de introducir cambios que resultasen en una gestión editorial más ágil y eficiente. Como parte de un proceso global de modernización de nuestra gestión, se analizó el antiguo proceso editorial de un manuscrito, cuya principal característica era el ser completamente manual. Hace una década el proceso original había sido reemplazado por un sistema basado en la recepción de los artículos en papel junto a una copia electrónica, la cual era distribuida vía correo electrónico a los evaluadores. Posteriormente se recibían los arbitrajes y se enviaban a los autores por el mismo medio computacional, los que después de efectuar los cambios solicitados, enviaban la segunda

Correspondencia a:

Francisco Cano

E-mail:fcano@med.uchile.cl 
versión a los editores en la misma forma que el primer manuscrito. El análisis de este sistema de trabajo detectó importantes aspectos que requerían ser modificados, entre los que destacaban el que los artículos y colaboraciones circulaban en su mayor parte en soporte papel; el seguimiento de un manuscrito resultaba altamente complejo e inefectivo; el sistema impreso no contaba con un método eficaz para alertar sobre los plazos cumplidos a los autores, evaluadores, editores, y -en general- a todos los actores de la edición. En general se pudo concluir que todo el proceso manual dependía en su funcionamiento de personas que individualmente debían controlar el ciclo en su conjunto. Así, el diagnóstico general del método se podría resumir en pocas líneas: cumplía su función, era totalmente manual, con gran dependencia de las personas involucradas, requería de mucho esfuerzo y era altamente falible.

Desde hace algunos años atrás, los más importantes medios editoriales científicos del mundo han adoptado sistemas como el Scholar One's Manuscript Central y el Open Journal System, sistemas de postulación de manuscritos on-line para su publicación. Los autores generan una presentación on line de su artículo (up-load), el cual es ingresado al sistema y puede ser monitoreado permanentemente durante el proceso de revisión por pares, quienes tienen acceso disponible para realizar su arbitraje con la máxima eficiencia. Estos sistemas on-line actualmente albergan a más de 400 publicaciones en el mundo, sin incluir a ninguna revista científica del área médica de nuestro país.

El año 2008 la Revista Chilena de Pediatría postuló al Concurso del Fondo de Publicación de Revistas Científicas de CONICYT con el Proyecto "Revista Chilena de Pediatria: de las publicaciones en papel a un proceso editorial on-line basado en Open Journal System". Habiendo sido favorecida con este Proyecto, durante el año 2009 se desarrolló un intenso trabajo por parte del Comité Editorial y un experto en informática, logrando generar exitosamente un sistema que permite automatizar el complejo proceso editorial de los artículos científicos. El nuevo método propuesto es similar al anterior en sus flujos y el esfuerzo de optimización fue dirigido fundamentalmente a la automatización de los procesos, hacerlos independientes de las personas y darles seguridad de funcionamiento. Para el correcto funcionamiento de todo el ciclo fue necesario seccionar las tareas creando perfiles específicos, y definiendo sus funciones principales: Gestor (Administrador General y Director), Editor, Editor de Sección, Revisor (o árbitro) y Autor. En el sistema, una persona puede tener asignado más de un perfil y al ingresar debe definir en cual quiere trabajar para tener acceso a las funciones específicas, pudiendo cambiar de perfil -dentro de la misma conexión- para tener acceso a otras. El investigador que hoy presente una contribución a nuestra Revista, debe en primer lugar ingresar a www.revistachilenadepediatria.cl, registrarse para generar el perfil de usuario, obtener un nombre de usuario y una contraseña, para luego iniciar proceso de subir (up-load) su artículo.

Dentro de los lineamientos de esta dirección editorial en la década, la integración y visualización de Revista Chilena de Pediatría participando activamente en grupos internacionales de edición de revistas biomédicas, ha sido un hito importante. La incorporación en 1999 de Chile al grupo de Editores de revistas de Sociedades de Pediatría del Cono Sur, formada por los equipos editoriales de las Revistas de las Sociedades de Pediatría de Argentina, Brasil, Bolivia, Paraguay, Uruguay y Chile. El trabajo de este grupo de editores ha permitido la consolidación de la edición de las revistas de la región de acuerdo a normas internacionales y la incorporación de todas ellas al sistema SciELO, ya sea en calidad de colección o como iniciativa en desarrollo. Por otra parte, la participación en la Agrupación Chilena de Revistas Biomédicas, desde 2005, ha permitido compartir experiencias editoriales y participar en la discusión de problemas comunes del trabajo editorial. La participación en estas agrupaciones, ha sido muy enriquecedor y ha permitido establecer redes de cooperación entre sus miembros y mostrar las fortalezas de las distintas revistas participantes.

Así hemos resumido los esfuerzos realizados por la actual Dirección de la Revista en la consolidación y modernización del proceso 
editorial, en el cual han participado activamente el Comité Editorial, que en forma desinteresada ha realizado una labor silenciosa y eficaz; el Directorio de la Sociedad Chilena de Pediatría, quien nos ha brindado un decidido apoyo en las distintas iniciativas emprendidas; CONICYT a cargo de quién en Chile se ha desarrollado el proyecto SciELO y muchos colaboradores que han contribuido con su esfuerzo a estos importantes logros con su permanente aporte científico.

Los resultados de las iniciativas emprendidas darán sus frutos en los años venideros, y la consolidación del proyecto de digitalización del proceso editorial, pondrá a Revista Chilena de Pediatría en una condición de liderazgo editorial en Chile, y constituirá una herramienta eficaz y segura para responder a la tarea de ser la principal publicación pediátrica de nuestro país. 